\title{
Eye-Safe Diode-Laser-Based Micropulse Differential Absorption Lidar (DIAL) for Water Vapor Profiling in the Lower Troposphere
}

\author{
AMin R. NEHRIR AND KEVIN S. REPASKY \\ Department of Electrical and Computer Engineering, Montana State University, Bozeman, Montana \\ JOHN L. CARLSTEN \\ Department of Physics, Montana State University, Bozeman, Montana
}

(Manuscript received 1 February 2010, in final form 20 September 2010)

\begin{abstract}
A second-generation diode-laser-based master oscillator power amplifier (MOPA) configured micropulse differential absorption lidar (DIAL) instrument for profiling of lower-tropospheric water vapor is presented. The DIAL transmitter is based on a continuous wave (cw) external cavity diode laser (ECDL) master oscillator that is used to injection seed two cascaded tapered semiconductor optical power amplifiers, which deliver up to $2-\mu \mathrm{J}$ pulse energies over a $1-\mu$ s pulse duration at $830 \mathrm{~nm}$ with an average power of $\sim 40 \mathrm{~mW}$ at a pulse repetition frequency of $20 \mathrm{kHz}$. The DIAL receiver utilizes a commercial 28-cm-diameter SchmidtCassegrain telescope, a 250-pm narrowband optical filter, and a fiber-coupled single-photon-counting Avalanche photodiode (APD) detector, yielding a far-field full-angle field of view of $170 \mu \mathrm{rad}$. A detailed description of the second-generation Montana State University (MSU) DIAL instrument is presented. Water vapor number density profiles and time-height cross sections collected with the water vapor DIAL instrument are also presented and compared with collocated radiosonde measurements, demonstrating the instruments ability to measure night- and daytime water vapor profiles in the lower troposphere.
\end{abstract}

\section{Introduction}

Water vapor is the most dominant greenhouse gas in the atmosphere (Trenberth et al. 2007). The radiative forcing for a clear sky due to water vapor is $75 \mathrm{~W} \mathrm{~m}^{-2}$, while for carbon dioxide $\left(\mathrm{CO}_{2}\right)$ it is a factor of 2 weaker at $32 \mathrm{~W} \mathrm{~m}^{-2}$ (Kiehl and Trenberth 1997). The sensitivity of radiative forcing due to a change in water vapor- $-\mathrm{CO}_{2}$ concentrations in the equatorial regions is small due to the already large greenhouse effect and therefore has a small direct impact on the reemitted downward infrared radiation. However, in the cold dry polar regions, the effects of a small increase in water vapor- $\mathrm{CO}_{2}$ caused by equatorial convective circulations are much greater (Trenberth et al. 2007).

Water vapor is also the dominant positive feedback mechanism in our climate system and a major reason why

Corresponding author address: Kevin S. Repasky, Dept. of Electrical and Computer Engineering, Montana State University, Cobleigh Hall, Rm. 610, Bozeman, MT 59717.

E-mail: repasky@ece.montana.edu temperature is so sensitive to changes in $\mathrm{CO}_{2}$. Unlike external forcings such as $\mathrm{CO}_{2}$, which can be added to the atmosphere, the level of water vapor in the atmosphere is a function of temperature. Water vapor is transported into the atmosphere via evaporation where the rate depends on the ocean and air temperature and is governed by the Clausius-Clapeyron relation. Atmospheric water vapor is directly related to the surface and atmospheric temperature and has been shown to have the largest positive feedback effect in the climate system (Trenberth et al. 2007).

Aerosols also play an important role in the earth's complex climate system. The increased aerosol loading of the atmosphere due to anthropogenic sources produces a negative radiative forcing that is similar in magnitude to the positive radiative forcing associated with the increase in anthropogenic greenhouse gases (Charlson et al. 1992). According to the Fourth Assessment Report (AR4) of the Intergovernmental Panel on Climate Change (IPCC), the radiative forcing due to aerosols currently has a "low level of scientific understanding" (Forster et al. 2007), resulting in the largest uncertainties in our 
understanding and modeling of the earth's climate system. The radiative forcing of aerosols depends on three coupled components of the climate system, including atmospheric aerosols, water vapor, and clouds. To better understand and model the role these atmospheric constituents play in the climate system, new observational instruments and techniques are needed to reduce these uncertainties (Forster et al. 2007). To better understand the aerosol indirect effect, Wulfmeyer and Feingold (2000) noted that lidar measurements of aerosol properties and water vapor can provide important information to enhance our understanding of the role of aerosols in the climate system.

Active remote sensors such as lidars have become an attractive alternative to passive sensors for monitoring the high spatial and temporal resolutions of atmospheric aerosols and water vapor. Various research groups around the world have developed and deployed both groundbased and airborne lidar systems to monitor vertical distributions of aerosols and water vapor simultaneously. Such instruments include Raman lidars (Goldsmith et al. 1998; Eichinger et al. 1999; Whiteman et al. 1998) and differential absorption lidars (DIALs; Browell et al. 1998; Rall et al. 1996; Wulfmeyer 1998; Wulfmeyer and Bosenberg 1998; Bosenberg 1998; Ehret et al. 1993; Ehret et al. 1998; Ismail et al. 2000; Wirth et al. 2009; Machol et al. 2004; Nehrir et al. 2009). Raman lidar instruments provide high spatial resolution of atmospheric water vapor by observing a characteristic Raman shift to longer wavelengths generated from inelastic backscattering in the atmosphere from high-power laser transmitters. Raman lidars have proven to be attractive for monitoring atmospheric processes because of their capabilities for monitoring multiple gas constituents simultaneously but suffer from high operating costs due to periodic instrument calibration as well as the need for bulky powerful laser transmitters required to overcome weak Raman scattering cross sections. Alternatively, the DIAL technique utilizes the elastic backscattered signal from a tunable laser transmitter to determine rangeresolved water vapor profiles. Because the DIAL technique uses a ratio of the return signals from a closely spaced pair of online and offline transmitter wavelengths, difficult calibration processes faced by Raman lidar instruments are avoided, making the DIAL technique a suitable method for ground-based and airborne mobile platform instruments used for long-duration aerosol and water vapor monitoring.

Large-scale water vapor and aerosol transport processes are difficult to observe from a single ground-based instrument, but can be easily monitored with high spatial and temporal resolution using airborne DIAL instruments at the expense of high operating and maintenance costs. The high spatial and temporal variability of water vapor can also be captured using an array of low-cost, eye-safe, automated DIAL instruments (Reagan et al. 1993). Water vapor transport processes used to enhance climate studies and weather forecasting capabilities can be measured using a network of commercial off-theshelf (COTS) built water vapor DIAL instruments that can also be supplemental to larger-scale airborne and satellite-based campaigns. Many high-power large-scale water vapor DIAL instruments have been successfully built and deployed that have been used for a wide range of sounding measurements in various geographical locations (Wulfmeyer 1998; Ismail et al. 2000), but few eye-safe autonomous systems have been successfully demonstrated. Recently, Nehrir et al. (2009) and Machol et al. (2004) have demonstrated the utility of using semiconductor lasers as DIAL transmitters. Diode lasers are extremely compact and inexpensive and can be widely tuned to access weak and strong water vapor absorption bands at 830 and $940 \mathrm{~nm}$, respectively. Recently, tapered semiconductor optical amplifiers (TSOAs) have become commercially available, allowing for eye-safe micropulse DIAL transmitters in the 830-nm spectral region.

The lidar group at Montana State University (MSU) is working toward the development of a ground-based autonomous eye-safe micropulse DIAL instrument for continuous water vapor and aerosol profiling in the lower troposphere. A first-generation water vapor DIAL instrument was discussed in detail earlier (Nehrir et al. 2009). A second-generation water vapor DIAL instrument has been developed based on pulsed semiconductor optical amplifiers. The second-generation DIAL transmitter makes use of the same diode-laser-based seed source as the first-generation DIAL but utilizes actively pulsed TSOAs to achieve peak pulse energies ranging from 1 to $2 \mu \mathrm{J}$, a factor of 10-20 greater in energy than the passively pulsed transmitter of the first-generation DIAL. The second-generation DIAL receiver has also been redesigned to utilize an extremely compact and light tight fiber coupling assembly, decreasing the overall ambient background noise of the DIAL instrument over the first-generation DIAL. The second-generation micropulse water vapor DIAL instrument based on a diode laser master oscillator power amplifier (MOPA) configured transmitter is described in this paper.

\section{Instrument description}

\section{a. DIAL laser transmitter}

A first-generation water vapor DIAL instrument has been developed at Montana State University (MSU), demonstrating the capabilities of diode-laser-based DIAL 


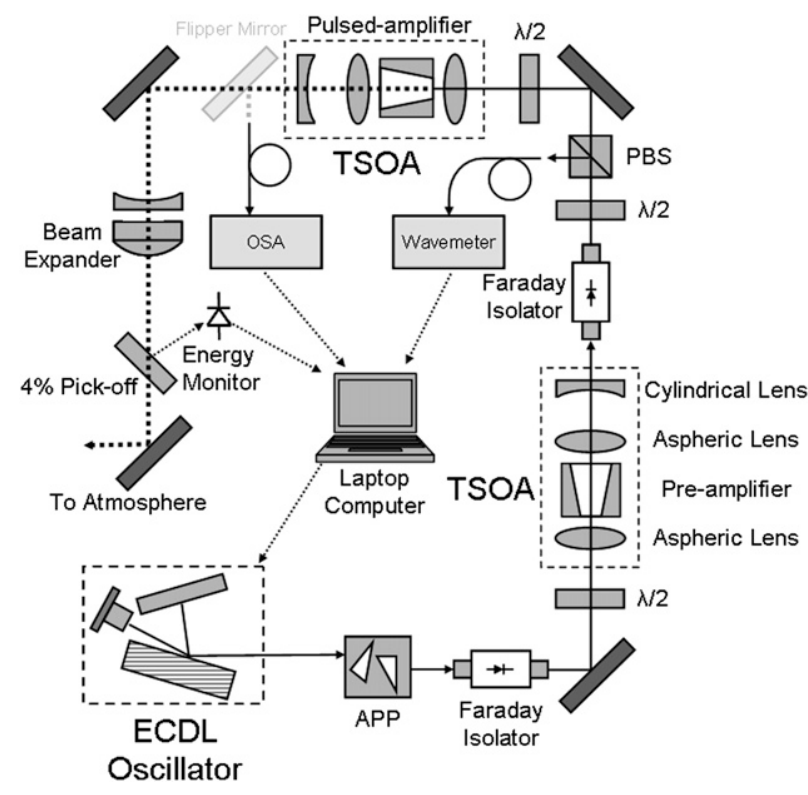

FIG. 1. Schematic of the MSU DIAL transmitter layout.

instruments (Nehrir et al. 2009). Low peak and average powers limited by commercially purchased TSOAs resulted in relatively long integration periods yielding moderate temporal resolution water vapor profiles up to $2.5 \mathrm{~km}$. A higher-power DIAL laser transmitter has been implemented in a second-generation water vapor DIAL to overcome the limitations set by the lower-power firstgeneration instrument.

A schematic of a second-generation water vapor DIAL transmitter is shown in Fig. 1. The entire secondgeneration DIAL instrument is housed on a $61 \mathrm{~cm} \times$ $122 \mathrm{~cm}$ optical breadboard. A widely tunable external cavity diode laser (ECDL), based on a Littman-Metcalf configuration (Nehrir et al. 2009), is capable of accessing the water vapor absorption band ranging from 824 to $841 \mathrm{~nm}$ is used as the seed source for the water vapor DIAL instrument. The output from a widely tunable ECDL in the Littman-Metcalf configuration has the narrow linewidth and broad tunability needed for the DIAL transmitter, but exhibits low continuous wave (cw) output power (Nehrir et al. 2009). To increase the optical power while maintaining the good spectral properties of the master laser, the ECDL is used to injection seed two custom-built cascaded TSOAs. To avoid vignetting on the input aperture of various optical components throughout the DIAL transmitter, the output from the ECDL is circularized using an anamorphic prism pair (APP). The beam-corrected output is then isolated using a 30-dB Faraday isolator to avoid unwanted feedback from the first-stage-tapered preamplifier from affecting the spectral properties of the master ECDL oscillator.

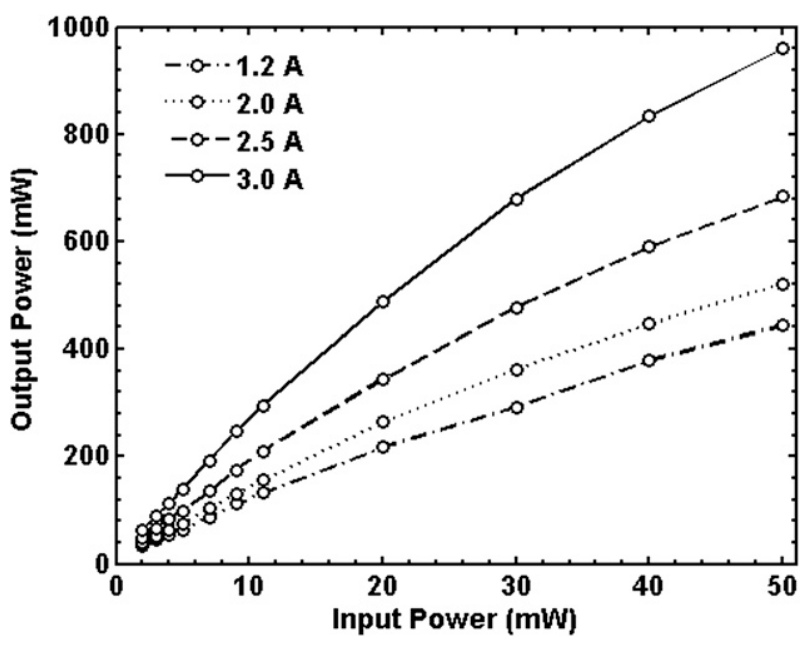

FIG. 2. The cw DIAL transmitter output power vs injection seeded power from the ECDL with cw injection currents ranging from 1.2 to $3.0 \mathrm{~A}$.

The output from the ECDL is used to injection seed a tapered semiconductor optical preamplifier (EYPTPA-830-01000) rated up to $1-\mathrm{W} \mathrm{cw}$. The polarization state of the output of the ECDL is aligned parallel to the junction plane of the first stage amplifier using a zeroorder half-wave plate. The custom-built tapered amplifiers comprise the two main sets of components: a strain released and thermally compensated brass housing for the c-mount TSOA and two modified commercially available three-axis fiber chucks that house the input and output coupling optics. A detailed description of the design and characterization of the TSOA housings are presented in Xiong et al. (2006).

The first-stage preamplifier is operated with a forward bias current of $1.2 \mathrm{~A}$, resulting in an increase of the $15-\mathrm{mW}$ cw output of the ECDL up to $50 \mathrm{~mW}$, yielding an overall gain of $5.2 \mathrm{~dB}$. The output from the preamplifier is isolated using a 30-dB Faraday isolator and is next incident on a second half-wave plate (HWP) and a polarizing beam splitter (PBS). Light rejected by the PBS is sent to a self-calibrating wavemeter (Burleigh$1500)$ to lock the seed laser's wavelength to the online, sideline, or offline wavelength of a water vapor absorption feature to within $\pm 88 \mathrm{MHz}$ (Nehrir et al. 2009). Differences between the measured wavelengths of the seed laser before and after the first stage amplifier are well below the resolution of the wavemeter and do not contribute to a bias of the DIAL transmitter's absolute wavelength measurement.

The light passed by the PBS is used to injection seed a second TSOA that is operated in saturation. A plot of the $\mathrm{cw}$ amplification of the second-tage TSOA for various drive currents is shown in Fig. 2. The temperatures 
of both amplifiers were stabilized to $15^{\circ} \mathrm{C}$. The input power to the second-stage TSOA was varied from 1 to $50 \mathrm{~mW}$ using a half-wave plate and a PBS for drive currents ranging from 1.2 to $3.0 \mathrm{~A}$. The ratio of the light passing through the PBS was controlled by rotating the wave plate. A small signal gain for $\mathrm{cw}$ seeding powers below $10 \mathrm{~mW}$ was measured to range from 12.43 to $14.66 \mathrm{~dB}$ for drive currents ranging from 1.2 to $3.0 \mathrm{~A}$, respectively. A saturated $\mathrm{cw}$ gain of $12.90 \mathrm{~dB}$ was measured for a 3.0-A forward-bias drive current, yielding approximately a $1-\mathrm{W}$ output for a $50-\mathrm{mW} \mathrm{cw}$ input seed power.

Pulsing of the DIAL transmitter is achieved by directly modulating the forward current to the secondstage TSOA. To evaluate the feasibility of an actively pulsed diode-based DIAL transmitter, a time domain study of the output pulse train was performed. A fast silicon photodiode was used to measure the peak output power of the DIAL transmitter over $1-\mu$ s pulse durations. Calibration of the photodetector was achieved by measuring and fitting the small signal $\mathrm{cw}$ responsivity of the photodiode to that of a National Institute for Science and Technology (NIST) traceable power meter. The temporal characteristics of the DIAL transmitter were studied by attenuating the output of the secondstage TSOA using an optical density (OD) 3.0 neutral density filter to avoid saturation affects and damage to the silicon photodiode.

The absolute maximum rating for the $\mathrm{cw}$ operating current of the TSOA as specified by the manufacturer is 3.0 A, yielding up to $1 \mathrm{~W}$ of output power with proper seeding. Higher peak powers have been obtained by overdriving the forward current to the TSOA in the pulsed regime. Low duty cycles and efficient conductive cooling allows for the excess heat generated by the overdriven TSOA to be dissipated between each laser shot. If the TSOA is not properly cooled, this technique of current pulsing can cause irreversible damage to the diode chip. The second-stage TSOA was pulsed using $1-\mu$ s current pulses at a pulse repetition frequency of $20.0 \mathrm{kHz}$, yielding a transmitter duty cycle of $2.0 \%$. A modulated forward current ranging from 3.0 to $5.5 \mathrm{~A}$ was supplied to the TSOA to study the peak power as well as the temporal evolution of an actively pulsed TSOA. A small cw bias current of $100 \mathrm{~mA}$ was also supplied to the TSOA to alleviate thermal straining of the gain medium when modulating the TSOA with high current pulses. The cw bias current was chosen to provide an extinction ratio of greater than 1000 between the $\mathrm{cw}$ leakage light and the amplified pulsed output. A plot of the peak output power of the DIAL transmitter as a function of time for two values of the pulsed forward current is shown in Fig. 3. Peak output powers of 0.95

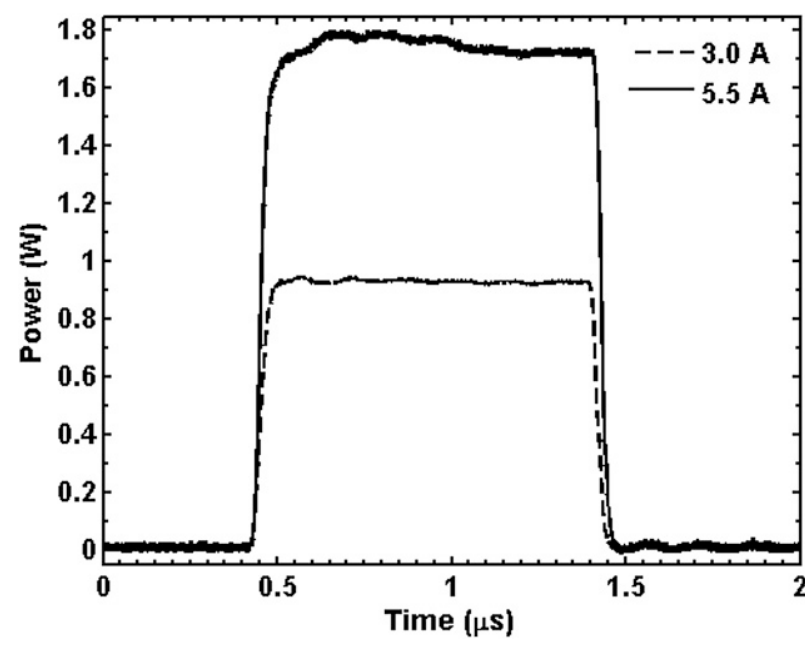

FIG. 3. Time dependence of the output power of the injectionseeded DIAL transmitter for 3.0- and 5.5-A current pulses. An increase in the output power of the DIAL transmitter is observed when the forward current to the second-stage amplifier is overdriven.

and $1.78 \mathrm{~W}$ were achieved with a $50-\mathrm{mW}$ input seed power over 1- $\mu$ s pulse widths for 3.0- and 5.5-A forward currents supplied to the TSOA, respectively. For output powers ranging from $\sim 1.0$ to $2.0 \mathrm{~W}$, rise times of less than $30 \mathrm{~ns}$ were observed. High-frequency impedance matching yields distinct square-shaped pulses with underdamped oscillations suppressed to less than $1.5 \%$ of the peak output power.

Higher peak powers achieved using larger pulsedforward currents have been observed in similar TSOAs at the increased risk of permanent damage to the input facet of the amplifier (Takase et al. 2007). For pulsedforward currents exceeding $6.5 \mathrm{~A}$, back reflections from the collimation optics as well as from the input polarizer of the optical isolator resulted in significant reverse amplification through the gain medium, causing irreversible damage to the input facet of the TSOA. Similar results were observed by Takase et al. (2007). The increased damage thresholds presented here over the damage thresholds presented by Takase et al. (2007) are thought to be a result of the anitreflection (AR) coatings of the collimation and isolation optics, as well as a more efficient decoupling method of the back-reflected light achieved by the slight misalignment of the DIAL transmitter optics. As a result, the optical isolator after the second-stage TSOA was removed to reduce the probability of reverse amplification, and a careful misalignment technique of the proceeding DIAL transmitter optics was used. This technique resulted in a $15 \%$ increase in the peak output power of the DIAL transmitter. 
The cascaded MOPA-configured DIAL transmitter can produce up to approximately $2.0 \mu \mathrm{J}$ of energy per pulse with a pulse repetition frequency of $20 \mathrm{kHz}$, yielding an average output power of approximately $40 \mathrm{~mW}$. After the second-stage TSOA, the 4.0-mm-diameter collimated beam is incident on a $5 \times$ beam expander, yielding a 20.0-mm-diameter output beam. The minimum divergence of the output of the DIAL transmitter was calculated to be approximately $0.1 \mathrm{mrad}$, where atmospheric turbulence such as scintillation has the potential to increase the divergence. The DIAL transmitter has a measured energy density of $0.636 \mu \mathrm{J} \mathrm{cm}^{-2}$ over a $1-\mu \mathrm{s}$ exposure time that is below the $0.91 \mu \mathrm{J} \mathrm{cm}^{-2}$ standard at $830 \mathrm{~nm}$ set by the American National Standards Institute (ANSI) for safe use of a laser. Since the 830-nm wavelength will not provide a natural aversion response such as a visible wavelength laser would, a 2-s exposure time is assumed. The corresponding maximum permissible exposure (MPE) per pulse for a 2-s exposure time is $45 \mu \mathrm{J} \mathrm{cm}^{-2}$. Hence, the MPE expressed as the cumulative exposure for the 2-s duration of the pulse train is $1.8 \mu \mathrm{J} \mathrm{cm}^{-2}$, which is below the $2.57 \mathrm{~mJ} \mathrm{~cm} \mathrm{~cm}^{-2}$ ANSI MPE standard, making the water vapor DIAL eye safe at a 0-m range from the instrument (ANSI 1993). Next, the beam-expanded output is incident on an AR-coated $4 \%$ wedged window. Light reflected from the wedged window is sent to a reference detector used to monitor the average output power of the DIAL laser transmitter while light passing through the wedged window is sent into the atmosphere coaxially with the DIAL receiver via a $45^{\circ}$ turning mirror. High temporal resolution energy monitoring of the DIAL transmitter was not necessary as the measured short-term shot-to-shot energy stability was better than $1.5 \%$.

Previously, Bosenberg (1998) described that errors due to laser properties resulting in a total measurement error of less than $3 \%$ are required for accurate DIAL measurements. These stringent laser properties arise due to the fact that the differential absorption cross section for the DIAL measurement of water vapor is highly dependent on the laser wavelength. For water vapor DIAL measurements in the near-infrared (NIR) portion of the spectrum, uncertainties in the laser linewidth, frequency stability, and spectral purity can lead to large errors in calculating the number density profile up through the lower troposphere. The requirements for the laser transmitter for a total measurement error of less than $3 \%$ from individual laser properties include a linewidth of less than $298 \mathrm{MHz}$, a spectral purity of greater than 0.995 , and a frequency stability of better than $\pm 160 \mathrm{MHz}$ (Bosenberg 1998). The operation of the diode-based MOPA laser with a narrow linewidth and a high spectral purity, and its operation at the center and side of the water vapor lines, ensures minimal influences in the DIAL measurements that are caused by the uncertainties in the laser spectral distribution.

A water vapor line selection criteria based on methods presented by Browell et al. (1991) was used to ensure minimal effects resulting from the sensitivity of water vapor absorption cross sections to uncertainty in the knowledge of the atmospheric temperature (Nehrir et al. 2009). The seed laser for the second-generation DIAL instrument is the same seed laser used for the firstgeneration DIAL instrument; hence, the laser linewidth and frequency stability measurements are expected to be the same as in Nehrir et al. (2009), which were stated previously as $0.300 \mathrm{MHz}$ and $\pm 88 \mathrm{MHz}$, respectively. The spectral purity measurement for the second-generation DIAL instrument is still measured to be $>99.5$. Because of the weak line strength of the selected water vapor absorption line, an updated saturated spectrum spectral purity measurement could not be obtained, so the previously stated lower limit of the spectral purity is assumed (Nehrir et al. 2009).

The spectral purity measurement described in Nehrir et al. (2009) was carried out by integrating and comparing the power spectral density measured across the full gain bandwidth of the semiconductor optical amplifier $(\sim 30 \mathrm{~nm})$ to that of the power spectral density measured across the bandwidth of the narrowband optical filter $(0.25 \mathrm{~nm})$. A 26-GHz-resolution spectrometer was used to make this measurement. The authors recognize that this technique for measuring the spectral purity of the DIAL transmitter is not extremely accurate. Work is on going to decrease the pulse repetition frequency of the DIAL transmitter such that an atmospheric spectral purity measurement using cirrus clouds at around $10-12 \mathrm{~km}$ can be obtained. A custom-built multipath $(>200 \mathrm{~m})$ gas absorption cell is also being considered to make a zero transmission spectral purity measurement. It is estimated that errors induced in the measurement by the lack of knowledge of the rangeresolved differential absorption cross section of water vapor, as well as the coarse frequency resolution of the wavemeter at the sideline wavelength, result in errors of less than $5 \%$ for boundary layer water vapor measurements. A summary of the second-generation water vapor DIAL transmitter specifications is shown in Table 1.

\section{b. DIAL receiver}

In addition to precise laser transmitter frequency control and high spectral purities required for tropospheric water vapor measurements, low-power DIAL instruments must also rely on highly efficient optical receiver assemblies to increase the signal-to-noise ratio (SNR) of the collected backscattered returns. For lidar instruments 
TABLE 1. Laser transmitter specifications for the diode-laser-based water vapor DIAL.

\begin{tabular}{ll}
\hline \hline Laser oscillator & $\begin{array}{c}\text { Littman-Metcalf external cavity } \\
\text { diode laser } \\
\text { Tapered semiconductor } \\
\text { optical amplifier }\end{array}$ \\
Laser amplifiers & $\begin{array}{c}\text { and } 828.287 \text { (offline) } \\
\text { Transmitted wavelengths } \\
\quad \text { (vacuum, nm) }\end{array}$ \\
PRF & $20.0 \mathrm{kHz}$ \\
Pulse length & $1.0 \mu \mathrm{s}$ \\
Short-term bandwidth & $\leq 0.3 \mathrm{MHz}$ \\
Long-term stability & $\pm 88 \mathrm{MHz}$ \\
Spectral purity & $>99.5 \%$ \\
Beam diameter & $\approx 20.0 \mathrm{~mm}$ \\
Beam divergence & $\approx 100 \mu \mathrm{rad}$ \\
\hline
\end{tabular}

targeted toward retrieving both near- and far-field backscattered returns with one receiving unit, a coaxial transmitter-receiver geometry is optimal (Harms et al. 1978). The schematic of the DIAL receiver is shown in Fig. 4. A 28 -cm f/10 Schmidt-Cassegrain telescope (Celestron CGE 1100) is used to collect the Rayleigh and Mie backscattered returns from the DIAL transmitter. To reduce the probability of stray light contamination at the detector, the light collected by the telescope is baffled using an adjustable 1-mm-diameter iris at the focal plane of the telescope. To reduce the needed detector size, or fiber optic core diameter in this case, a field lens placed at the focal plane of the telescope is used to reimage the off-axis backscattered light on to the detector plane. Although increasing the solar background noise at the detector, the field lens allows for the single-channel DIAL receiver to more efficiently collect the near-field returns where important water vapor dynamics are present. The effects of the increase in the skylight background noise due to the field lens in the far field are corrected by subtracting off a prepulse background noise measurement on a second-to-second basis.

Next, the backscattered light is collimated to a nominal diameter of $4.25 \mathrm{~mm}$ using a $50.0-\mathrm{mm}$ converging lens. The collimated light is then spectrally filtered using a 0.25 -nm full width at half maximum (FWHM) bandpass filter (BARR Associates) with a $65 \%$ transmission at a center wavelength of $828.06 \mathrm{~nm}(828.187 \mathrm{~nm})$ in air (vacuum). The filtered light is then incident on an AR-coated $45^{\circ}$ turning mirror used for coarse alignment of the DIAL receiver. Next, the light is fiber coupled into a shielded multimode optical fiber with a core diameter of $105 \mu \mathrm{m}$ and a numerical aperture (NA) of $\mathrm{NA}=0.22$ using an $11.0-\mathrm{mm}$ focal length commercially available six-axis micropositioning fiber coupling stage (Thorlabs PAF-X-11-PC-B). The entire receiver module is assembled using a series of lighttight Thorlabs SM1 lens

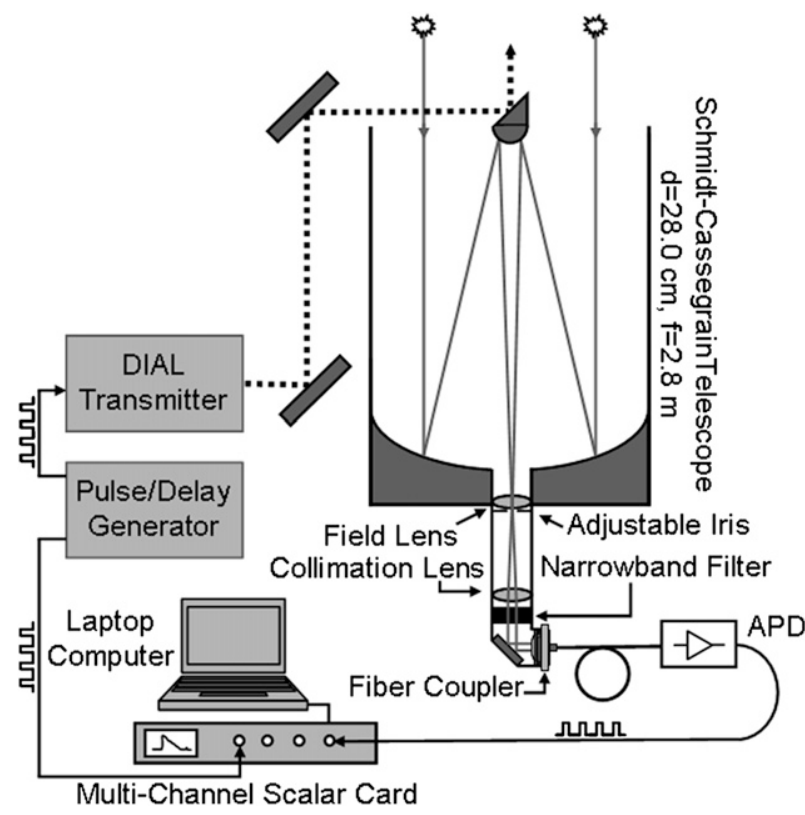

FIG. 4. Schematic of the DIAL receiver layout. All receiver optics are attached directly to the telescope and housed in a lighttight 1-in. tube assembly.

tubes to ensure low background noise at the detection stage.

To further increase the SNR of the MSU water vapor DIAL instrument during both daytime and nighttime operation, a narrow field-of-view receiver was designed and implemented to suppress unwanted solar and lunar background radiation. The $105-\mu \mathrm{m}$-diameter multimode fiber used to collect the backscatter light was designed to act as the system field stop, yielding an extremely narrow full-angle field of view of $37.5 \mu \mathrm{rad}$ when using a $28.0-\mathrm{cm}$-diameter $\mathrm{f} / 10$ telescope. Projection of the backscattered light imaged at the telescope's focal plane onto the multimode fiber through the collimation and fiber coupling optics yields a $4.5 \times$ magnification of the field of view of the DIAL receiver, resulting in a fullangle field of view of $170 \mu \mathrm{rad}$. The final receiver fieldof-view design of $170 \mu \mathrm{rad}$ was a compromise between sufficient background noise suppression and overlap walk-off between the transmitter and receiver due to thermal drifts of the transmitting optics.

The collected backscattered light is detected with an avalanche photodiode (APD) detector (Perkin Elmer SPCM-AQRH-13-FC) that is operated in Geiger mode. The output of the APD provides $>1.0 \mathrm{~V} 15$-ns-wide transistor-to-transistor logic (TTL) pulses for every photoelectron created at the detector junction plane. A specified APD dark count rate of less than 250 photoelectron counts per second and a relatively high quantum efficiency of $45 \%$ at $830 \mathrm{~nm}$ results in high 
TABLE 2. Optical receiver specifications for the MSU water vapor DIAL.

\begin{tabular}{ll}
\hline \hline Telescope & Schmidt-Cassegrain \\
Primary mirror diameter & $28.0 \mathrm{~cm}$ \\
Full-angle field of view & $170 \mu \mathrm{rad}$ \\
Detector & Si photon-counting APD \\
APD quantum efficiency & $45 \%$ \\
Optical filter bandwidth & $0.25 \mathrm{~nm}$ \\
Receiver efficiency & $\approx 10.0 \%$ \\
Digitizer bandwidth & $20.0 \mathrm{MHz}$ \\
Range resolution & $150 \mathrm{~m}$ \\
Total integration period & $10-30 \mathrm{~min}$ \\
\hline
\end{tabular}

SNRs of the DIAL returns up through the planetary boundary layer $(\sim 500 \mathrm{~m}-4.0 \mathrm{~km})$ with sufficient spatial and temporal averaging. Furthermore, due to a short APD dead time of $32 \mathrm{~ns}$ (below $5 \mathrm{Mc} \mathrm{s}^{-1}$ ) and an extremely low after-pulsing probability of $0.5 \%$, gating of the APD is not necessary and is therefore allowed to operate in a free running mode. A linear operating bandwidth of approximately $1 \mathrm{Mc} \mathrm{s}^{-1}$ yields a high dynamic range, allowing for the collection of near- and farfield returns to be obtained with one detector. DIAL returns ranging between 1 and $15 \mathrm{Mc} \mathrm{s}^{-1}$ require a linear correction factor ranging between 1.08 and 8 , respectively. Signal strengths exceeding $10 \mathrm{Mc} \mathrm{s}^{-1}$ are assumed to be saturated and are discarded. A summary of the MSU water vapor DIAL receiver parameters is shown in Table 2.

\section{c. Data acquisition and postprocessing}

The light sampled by the APD is monitored using a 20-MHz four-channel multichannel scalar card (MCS) data acquisition system (Sigma Space Corporation) and is triggered by a digital delay/pulse generator. The 1- $\mu \mathrm{s}$ pulse corresponding to a 150 -m-range resolution is oversampled by the MCS, yielding 20 data points per $150 \mathrm{~m}$, corresponding to a sampled vertical range resolution of $7.5 \mathrm{~m}$. The summing of these $7.5-\mathrm{m}$ bins is performed during postprocessing where 20 bins are grouped together to yield a $150-\mathrm{m}$ range resolution for the DIAL measurement. The range-corrected total backscatter measurements utilized the $7.5-\mathrm{m}$ range resolution set by the MCS but still exhibit a 150-m ambiguity in the range of the scattering events.

The operation and automation of the water vapor DIAL instrument is controlled via the Labview programming environment using a portable laptop computer and is described in detail in Nehrir et al. (2009). Active locking of the DIAL transmitter on to and off of the water vapor absorption line is achieved using a voltage-controlled feedback loop to the master ECDL's piezo-electric transducer (PZT) using Labview. The DIAL instrument is autonomously tuned to a user-defined online or sideline wavelength using a self-calibrating Burleigh wavemeter to monitor the DIAL transmitter's wavelength to within $\pm 88 \mathrm{MHz}$, where data are then obtained for $60 \mathrm{~s}$. The DIAL transmitter's wavelength is then tuned to an offline wavelength where data are once again taken for $60 \mathrm{~s}$ absent of water vapor absorption. Prior to each laser pulse, $5 \mu$ s of background data are obtained for postprocessing noise reduction. This results in a maximum resolvable DIAL return altitude of $6.0 \mathrm{~km}$. Also, a 1-2-s settling time between the online and offline wavelengths is provided to allow the wavelengthselective PZT to settle out to its steady state value. This process is repeated for a total of 10-30 min depending on the atmospheric conditions in order to obtain enough backscattered returns to calculate an accurate water vapor number density profile up through the lower troposphere.

Recently, sideline tuning of the water vapor absorption line has been exploited in order to access weaker absorption cross sections. Sideline tuning of the DIAL transmitter diminishes line saturation affects at lower altitudes and allows for higher SNRs at higher altitudes, allowing for more accurate water vapor number density retrievals beyond the boundary layer for different atmospheric conditions using one water vapor absorption line. The data presented throughout the remainder of this paper were obtained using sideline tuning of the 828.187-nm (vacuum) line to $828.195 \mathrm{~nm}$ (vacuum). Once the backscattered returns have been collected by the receiver and sampled by the MCS, the Matlab programming environment is used to analyze the data and calculate both water vapor number density profiles as well as timeheight plots of water vapor number densities using the DIAL equation (Kovalev and Eichinger 2004).

\section{Experimental results}

Micropulse DIAL instruments such as the water vapor DIAL that is deployed at MSU exploit high pulse repetition frequencies, and spatial and temporal averaging to obtain reasonable SNRs such that number density or mixing ratio measurements of the particulates of interest can accurately be calculated. The power of MSU's DIAL instrument lies in its ability to measure range- and time-resolved water vapor number densities up to and in some cases beyond the planetary boundary layer given a clear atmosphere. Several case studies under varying atmospheric conditions demonstrating the wide range sounding capabilities of the diode based DIAL instrument are presented.

\section{a. Nighttime measurements}

Initial testing of the second-generation ground-based MSU DIAL instrument was performed during a small 


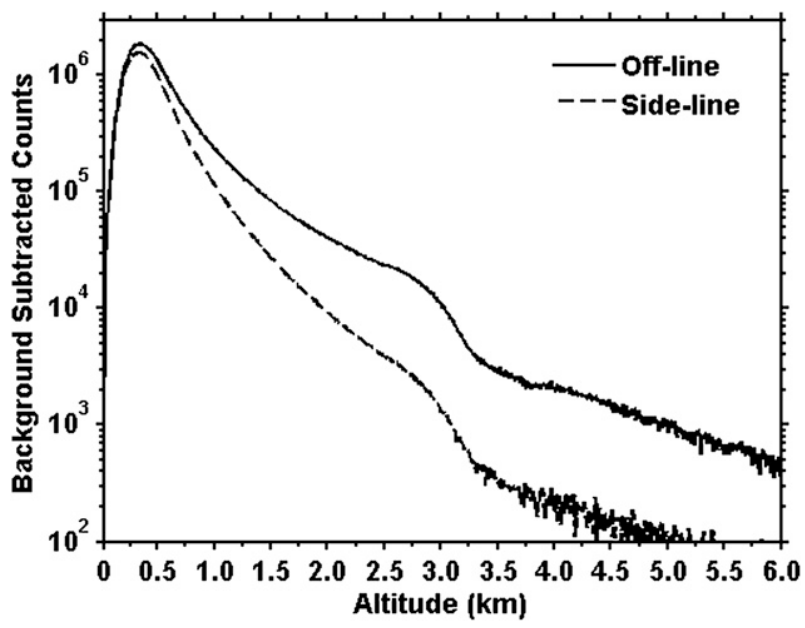

FIG. 5. Log-linear plot of the background subtracted sideline and offline atmospheric backscattered returns for a 20 -min $(10 \mathrm{~min}$ sideline and $10 \mathrm{~min}$ offline) total integration period centered around 0615 UTC (0015 MDT) on 11 Aug 2009. A 7.5-m vertical range resolution yields appreciable backscattered returns at the sideline wavelength up to the top of the planetary boundary layer and up to $6.0 \mathrm{~km}$ for the offline wavelength, well into the free troposphere.

nighttime campaign in order to maximize the instrument performance, which would otherwise be limited by the daytime background solar radiation. Initial measurements with the DIAL instrument were conducted during 10-11 August 2009 where the evolution of a stable nocturnal boundary layer was observed over approximately an 8 -h period. To begin assessing the nighttime performance of the instrument, the water vapor DIAL was operated for a 20-min period centered at 0615 UTC [0015 mountain daylight time (MDT)] on 11 August 2009 where 10 min of sideline-tuned backscattered data and 10 min of offline-tuned backscattered data were recorded in 60-s interleaved increments as discussed in the data acquisition section earlier.

A plot of the background-subtracted photon counts as a function of altitude for a 20-min total integration period for the sideline and offline wavelengths is shown in Fig. 5. The dashed and solid lines represent the sideline and offline atmospheric backscattered photoelectron counts measured by the DIAL instrument, respectively. A large dynamic range of $\sim 10^{4}$ from the initial overlap at $0.3-0.5 \mathrm{~km}$ up to $6.0 \mathrm{~km}$ due to the sensitivity of photon-counting APDs can be seen in the backscattered returns for both the sideline and offline wavelengths where the attenuation at the sideline wavelength is attributed to water vapor absorption. A decrease in the backscattered returns at approximately $3.0 \mathrm{~km}$ can be seen at both DIAL wavelengths due to confinement of the aerosols trapped below the planetary boundary layer
(PBL). Rapid degradation of the backscattered signal above the PBL into the free troposphere is attributed to two main causes. First, a significant fraction of the transmitted light through the PBL has been attenuated as a result of forward and backward scattering due to high levels of aerosol loading from regional biomass burning, resulting in low laser energies for scattering in the free troposphere. Second, the backscattered signals at both DIAL wavelengths begin to degrade rapidly beyond the PBL due to the lack of aerosol scattering in the free troposphere, causing the SNR of the instrument to decrease exponentially beyond the PBL and hence limiting the maximum resolvable altitude for water vapor profiles up to $\sim 3.8 \mathrm{~km}$ where the SNR of the sideline returns drops below an acceptable level (Wulfmeyer and Walther 2001).

The nighttime collected backscattered returns shown in Fig. 5 are used with radiosonde-derived temperature and pressure profiles to arrive at a water vapor number density profile using the DIAL equation (Nehrir et al. 2009; Kovalev and Eichinger 2004). Although a temperature-insensitive water vapor absorption line was chosen using methods presented by Browell et al.(1991) (Nehrir et al. 2009), the differential absorption cross section of water vapor is still calculated as a function of altitude using radiosonde-derived temperature and pressure profiles to reduce the error in the water vapor number density measurement. In the absence of radiosonde measurements, modeled temperature and pressure profiles using surface temperature and pressure measurements are calculated up through the lower troposphere (Machol et al. 2004). Using the range-dependent sideline and offline absorption cross sections together with the background subtracted atmospheric returns shown in Fig. 5, the DIAL equation can be used to arrive at a water vapor number density profile as a function of altitude, as shown in Fig. 6. Using 150-m spatially averaged range bins and a 20 -min (10 min sideline, 10 min offline) integration period, the water vapor number density profile retrieved using the DIAL instrument (solid black line) shows good agreement with the collocated in situ radiosonde measurement (dashed gray line) from near the surface up to the top of the boundary layer. Full overlap between the DIAL transmitter and receiver is achieved at approximately $500 \mathrm{~m}$ above the ground. The nighttime measurements shown in Fig. 6 exhibit an approximately $5 \%$ error relative to the radiosonde measurement from $0.5 \mathrm{~km}$ above the ground-based instrument up to $\sim 3.7 \mathrm{~km}$. The error bars in Fig. 6 were calculated by using the variance of the backscattered returns. An increase in the overall measurement error due to a combination of signal-induced noise, electronic noise, and background noise can be seen at altitudes above the 


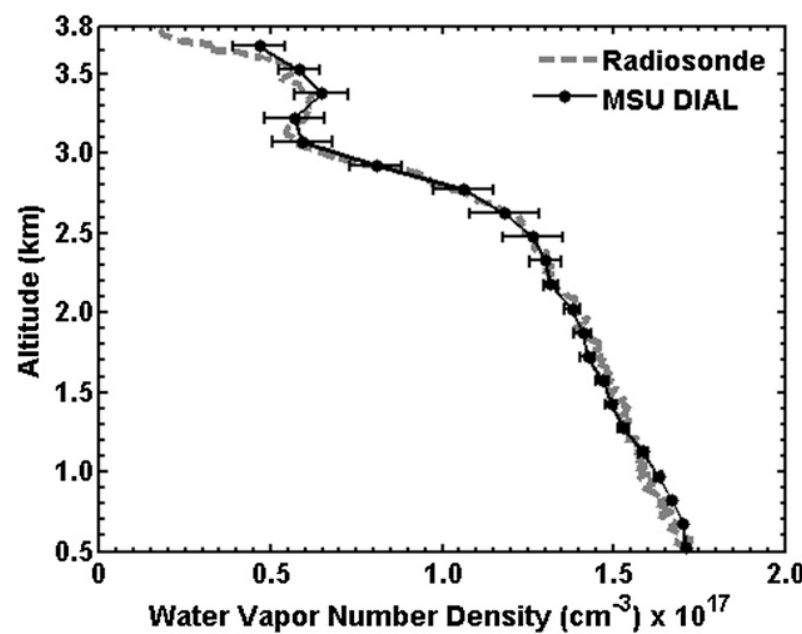

FIG. 6. Vertical water vapor number density profile recorded starting at 0605 UTC (0005 MDT) on 11 Aug 2009 using the atmospheric returns obtained from Fig. 5. A 20-min total integration period and 150-m down-sampled vertical range resolution are displayed.

boundary layer at $\sim 2.5 \mathrm{~km}$ where significantly fewer aerosol scattering events occur.

The utility of MSU's narrow field-of-view high pulse repetition frequency (PRF) DIAL instrument lies in its ability to measure range- and time-resolved water vapor number densities up through the lower troposphere with relatively short integration periods given clear atmospheric conditions. Nighttime false-color plots of the atmospheric backscatter and the associated water vapor number density cross sections over an 8-h extended period on 10 August 2009 are shown in Figs. 7a and 7b, respectively. Figure 7 a represents a time-height cross section of the range-corrected backscattered returns using the offline data collected with the water vapor DIAL instrument. Although not calibrated, the offline returns provide an insight into the atmospheric aerosol backscatter. A 1-s integration period and 7.5-m vertical range resolution provide high temporal and spatial resolutions for monitoring a stable nocturnal boundary layer. A well-defined boundary layer at $3.0 \mathrm{~km}$ is visible throughout the evening due to aerosol loading from regional biomass burning. Mixing of the aerosols at the top of the boundary layer can be seen early in the evening from approximately 0345 (2145) until 0715 UTC (0115 MDT) due to the residual convective heating from the prior day. A discontinuity in the atmospheric backscattered returns at approximately 0520 UTC (2320 MDT) was caused by an accidental collision with another lidar instrument that was being repositioned. Although clearly visible in the offline range-corrected backscattered returns, instantaneous discontinuities such as the one in Fig. 7a do not affect the number density measurement due to the differential normalization of the data collected between the online and offline wavelengths.

Water vapor number density cross sections associated with the atmospheric backscatter presented in Fig. 7a from 10 August 2009 are shown in Fig. 7b. The 10-minaveraging times for both the online and offline wavelengths were utilized to create one range-resolved water vapor number density profile calculated using the DIAL equation (Nehrir et al. 2009; Kovalev and Eichinger 2004). Water vapor number density time series are then derived by using a 20 -min running-average window (10 min of online and 10 min of offline data) of the backscattered returns through the DIAL equation across the full dataset. A nearest-neighbor interpolation algorithm is then used to smooth the data in space and time to increase the viewing resolution of the water vapor time series to $25 \mathrm{~m}$ in the vertical direction and $3.33 \mathrm{~min}$ in the horizontal direction. This method will be referred to as a profile interpolation. With 20-min total averaging times and an up-sampled range and temporal resolution, microscale convective features that agree well with in situ measurements can be seen up through the boundary layer to the free troposphere. A collocated radiosonde measurement taken starting at 0615 UTC [0015 mountain daylight time (MDT)] as indicated by the black dotted line in Fig. 7 is shown in Fig. 6. As previously discussed, the DIAL measurements show good agreement with the in situ radiosonde measurements up through the lower troposphere, demonstrating the capabilities of the DIAL instrument for water vapor number density retrievals up through the lower troposphere. The high spatial and temporal resolutions of Figs. $7 \mathrm{a}$ and $7 \mathrm{~b}$ depict weak remnants of a mixed layer from the residual convective heating of the surface from the prior day, which is evident by the transport of aerosols and warm moist air to the top of the boundary layer early in the evening. Furthermore, as the evening progresses under clear skies, the convective thermals begin to cease as the radiation into the atmosphere cools the surface, which results in the cooling of the neighboring air mass through molecular conduction, hence allowing the turbulence to decay into the mixed layer from the previous day. This settled mixed layer, which contains the remaining moisture, convected heat, and aerosols from surrounding biomass burning, is known as the residual layer and can be seen on average from approximately 1.0 to $2.6 \mathrm{~km}$ from the water vapor number density cross sections presented in Fig. $7 \mathrm{~b}$. By the end of the evening, the residual layer is largely trapped between a stratified shallow nocturnal boundary layer near the ground and a capping inversion at the top of the previous day's entrainment zone. The statically stable 


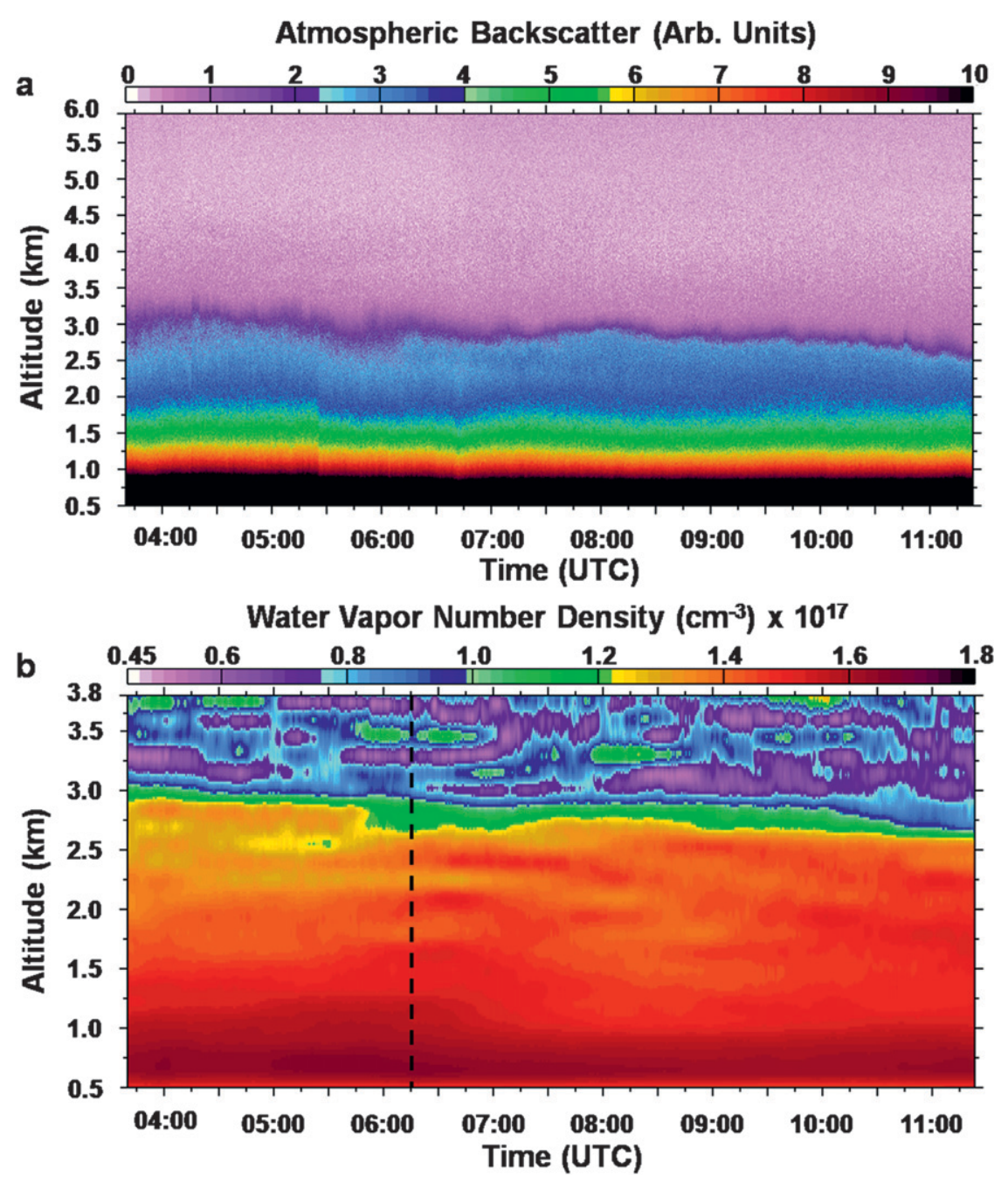

FIG. 7. (a) Vertical time series of the nighttime offline range-corrected backscattered returns for 10-11 Aug 2009. A 7.5-m vertical range resolution and 1-s temporal resolution are displayed. (b) Vertical time series of nighttime water vapor number densities measured during 10-11 Aug 2009. A 25-m vertical range resolution and 3.33-min temporal resolution are displayed. A collocated radiosonde measurement shown in Fig. 6 is indicated by the black dotted line at 0615 UTC.

nocturnal boundary layer seen in the lowest $1.2 \mathrm{~km}$ of the troposphere is formed as the cold nighttime surface cools the air mass near the ground, converting the bottom of the residual layer into a growing stable nocturnal layer. The capping inversion depicted by the green stratified layer at approximately $2.75 \mathrm{~km}$ in Fig. $7 \mathrm{~b}$ is formed by warm air convecting to the top of the boundary layer in the early evening, which is then trapped between the cooler free-tropospheric air and the cooling residual mixed layer. As the evening progresses into the following morning, the capping inversion gradually falls and diminishes as the surface cools off and the nocturnal boundary layer grows.

\section{b. Daytime measurements}

Monitoring the microscale dynamics of an evolving planetary boundary layer from a stable stratified nocturnal boundary layer at night into an unstable convective mixing state during the daytime provides useful insights toward gaining a better understanding of fundamental atmospheric transport processes. To assess the daytime performance as well as the long-term operating functionality of the MSU DIAL instrument, it was operated continuously over the course of 3 days where the atmospheric backscatter and water vapor cross sections were monitored and compared to in situ collocated 


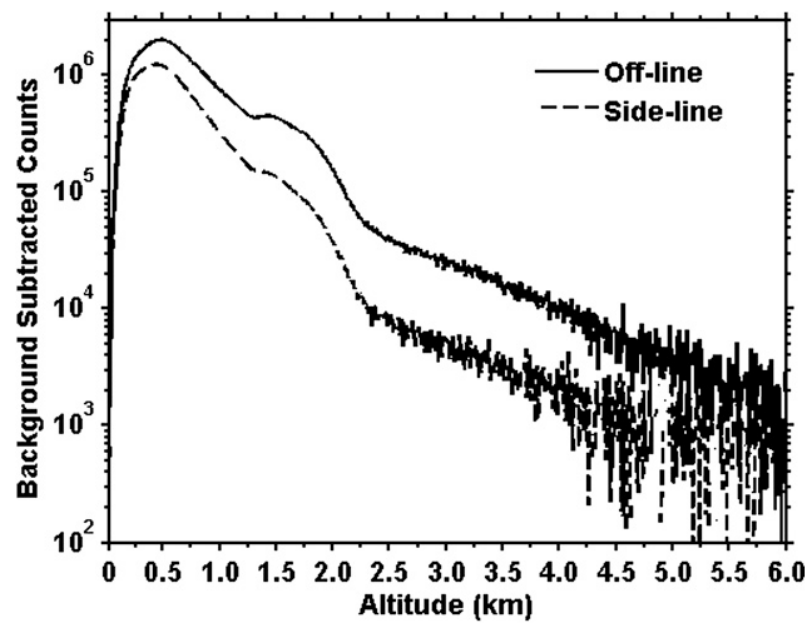

FIG. 8. Log-linear plot of the daytime background subtracted sideline and offline atmospheric backscattered returns for a 30-min (15 min sideline and 15 min offline) total integration period centered around 1435 UTC (0835 MDT) on 11 Sep 2009. A 7.5-m vertical range resolution yields appreciable backscattered returns up to the top of the planetary boundary layer around $2.0 \mathrm{~km}$, where noise from solar background radiation then dominates the returns.

radiosonde measurements. The DIAL instrument was operated continuously starting from 1435 UTC (0835 MDT) on 11 September 2009 until 0245 UTC (2045 MDT) on 13 September 2009. The initial daytime performance of the DIAL instrument was studied by analyzing the SNR of the raw atmospheric backscattered returns. Plots of the daytime background-subtracted photon counts as of function of altitude for a 30-min total integration period starting at 1420 UTC for the sideline and offline wavelengths are shown in Fig. 8. A range resolution of $7.5 \mathrm{~m}$ limited by the 20-MHz MCS data acquisition system and 15-min sideline and 15-min offline integration periods yield relatively good SNR up to the top of the planetary boundary layer at approximately $2.3 \mathrm{~km}$, where the sideline returns then become dominated by noise due to attenuation of the signal from water vapor absorption. Spatial averaging of the backscattered returns up to 150 -m range bins increases the SNR of the instrument and allows for daytime time water vapor number density profiles to be retrieved up to the free troposphere. Plots of a daytime water vapor number density profile as function of altitude using a 30-min total integration period centered at 1435 UTC (0835 MDT) and the associated water vapor time-height cross sections for a 1-h period are shown in Figs. 9a and 9b, respectively. Good agreement between the $150-\mathrm{m}$ spatially averaged DIAL measurements and the data collected with the collocated in situ radiosonde measurement can be seen from 0.5 to $2.5 \mathrm{~km}$ in Fig. 9a. Drag at the ground causing near-surface resistance of strong positive (westerly) zonal winds resulting in a distinct wind shear layer can be seen at approximately $2.25 \mathrm{~km}$. A strong negative gradient in the water vapor number density at the shearing layer can be seen where the relative humidity $(\mathrm{RH})$ calculated from the radiosonde measurements dropped from approximately $54 \%$ at $2.0 \mathrm{~km}$ to less than $4.0 \%$ at $2.3 \mathrm{~km}$. An overall error of less $14 \%$ between the daytime water vapor number densities measured using the DIAL instrument and the in situ radiosonde measurements was measured form $0.5 \mathrm{~km}$ up to approximately $2.0 \mathrm{~km}$. Deviations between the two measurements up to the top of the boundary layer are attributed to the differences in the sampled air masses between the DIAL and radiosonde measurements due to the strong westerly winds. Due to the lower concentration of aerosols, low measured relative humidity, and relatively large solar-induced background noise, the errors between the in situ measurements and the DIAL measurements above the boundary layer increase to approximately $50 \%$.

Although the maximum altitude for an acceptable accuracy of less than $10 \%-15 \%$ for the DIAL measurement is limited to the top of the boundary layer for daytime measurements, qualitative information can be gathered about the spatial and temporal distributions of water vapor past the planetary boundary layer into the free troposphere. Water vapor number density cross sections associated with the water vapor profile presented in Fig. 9a from 11 September 2009 are shown in Fig. 9b. The 30-min (15 min sideline, $15 \mathrm{~min}$ offline) integration periods and up-sampled range and temporal resolution using profile interpolation reveal the evolution of a short-term daytime inversion as well as a sustained shearing layer that agrees well with in situ measurements. A collocated radiosonde measurement taken starting at 1435 UTC (0835 MDT) shown in Fig. 9a is indicated by the black dotted line in Fig. $9 \mathrm{~b}$. The dry inversion layer up around $1.8 \mathrm{~km}$ at the beginning of the dataset begins to mix in with the turbulent boundary layer and diminishes at approximately 1500 UTC (0900 MDT). The shearing layer, evidenced by the negative water vapor gradient at approximately $2.25 \mathrm{~km}$, continues throughout the duration of the measurement.

\section{c. Long-duration boundary layer measurements}

Given the individual daytime and nighttime performances of the MSU DIAL instrument presented in Figs. 5-9, long duration datasets were thought to be achievable with integration periods below those of the lifetimes of typical tropospheric microscale features. To assess the long-term performance of the DIAL instrument, extended periods of clear skies were required such that relative atmospheric backscatter and water vapor 
a
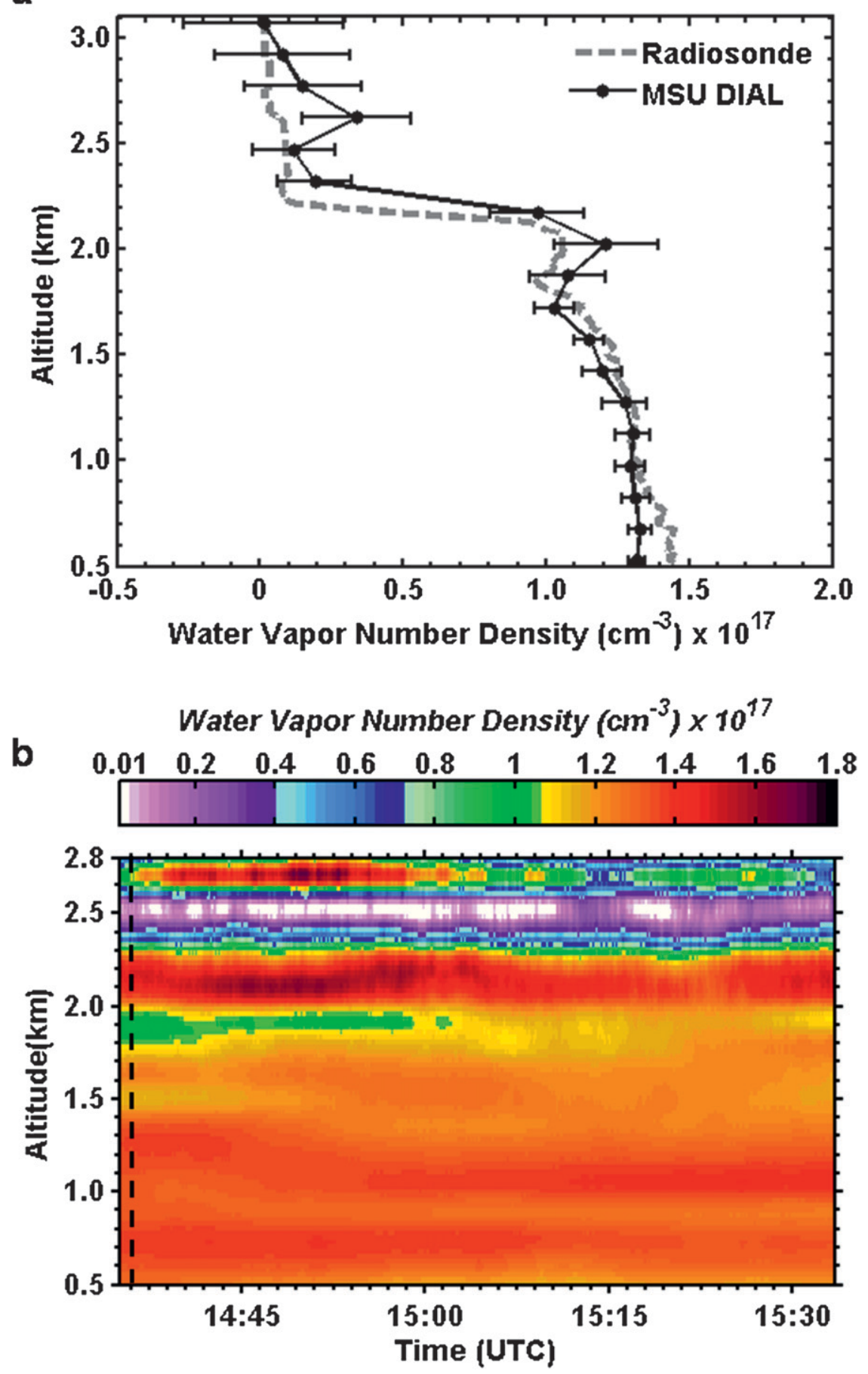

FIG. 9. (a) Vertical daytime water vapor number density profile recorded starting at 1420 UTC (0820 MDT) 11 Sep 2009 using the atmospheric returns obtained from Fig. 8. A 30 -min total integration period and $150-\mathrm{m}$ vertical range resolution are displayed. Good agreement between the DIAL measurements and the collocated in situ measurements can be seen from near the surface up to the top of the boundary layer. (b) Vertical time series of daytime water vapor number densities measured during the morning of 11 Sep 2009 showing the evolution of a daytime shearing layer at the top of the planetary boundary layer. A 25-m vertical range resolution and 3.33-min temporal resolution are displayed. A collocated radiosonde measurement shown in (a) is indicated by the black dotted line at 1435 UTC. 

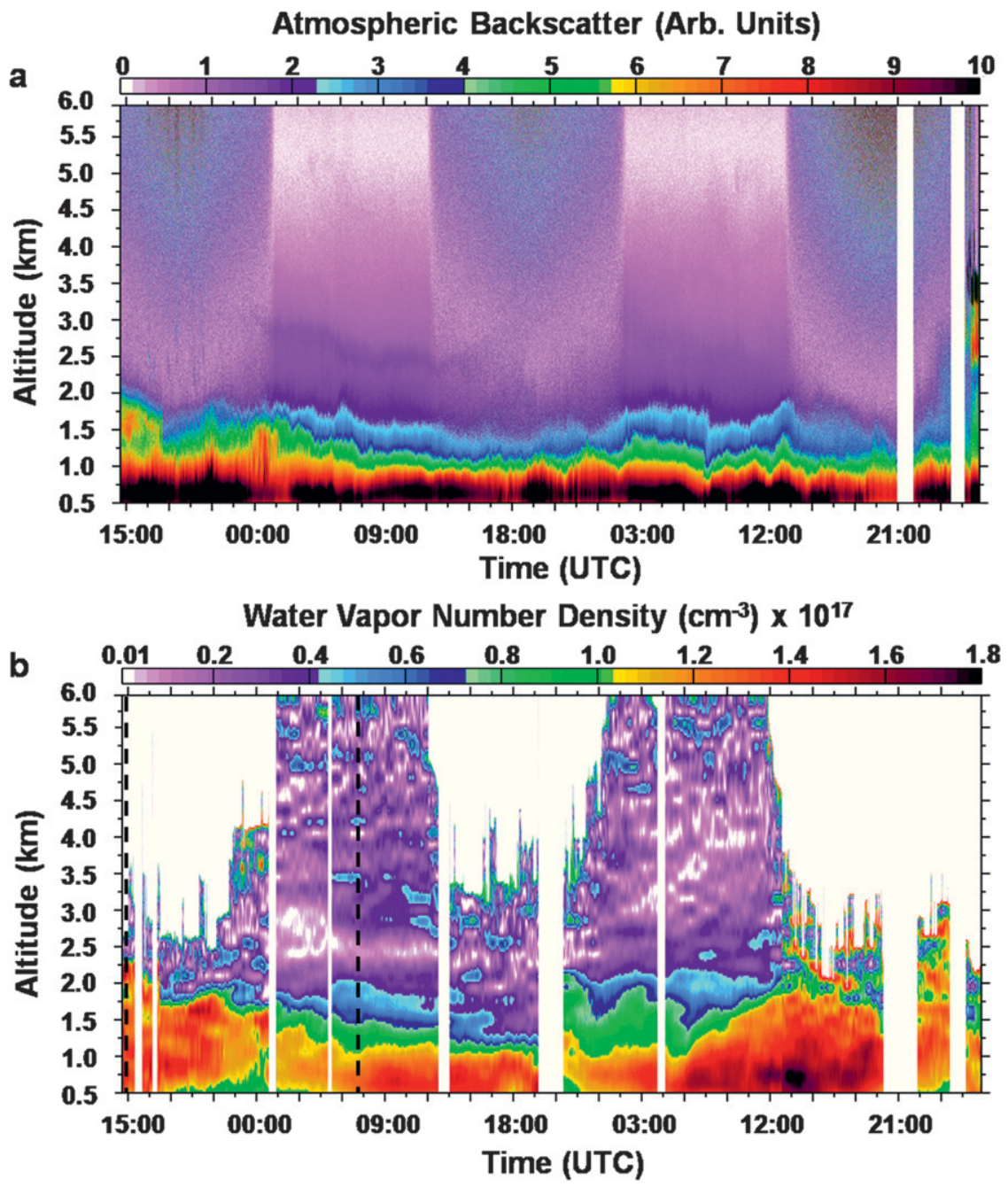

FIG. 10. (a) Vertical time series of the offline range-corrected backscattered returns for 11-13 Sep 2009. A 7.5-m vertical range resolution and 1-s temporal resolution are displayed. (b) Vertical time series of nighttime water vapor number densities measured during 11-13 Sep 2009. A 25-m vertical range resolution and 3.33-min temporal resolution are displayed. Two collocated radiosonde measurements indicated by the black dotted lines at 1435 UTC 11 Sep 2009 and 0700 UTC 12 Sep 2009 are shown in Figs. 9a and 12, respectively, demonstrating the daytime and nighttime capabilities of the MSU DIAL instrument.

number densities could be recorded up through the planetary boundary layer. The DIAL instrument was autonomously operated over a 60 -h period where diurnal water vapor number densities and atmospheric backscatter were monitored. False-color plots of the atmospheric backscatter and the associated water vapor number density cross sections over the 60 -h extended period starting from 1420 UTC on 11 September 2009 are shown in Figs. 10a and 10b, respectively. A 1-s integration period and $7.5-\mathrm{m}$ vertical resolution were used for the atmospheric backscatter shown in Fig. 10a. A profile interpolation of a sliding 30-min (15 min sideline, $15 \mathrm{~min}$ offline) integration period used to calculate the tropospheric water vapor content yields 3.33-min temporal resolution and 25-m vertical resolution for the water vapor cross sections shown in Fig. 10b. White omitted areas in Figs. 10a and 10b are attributed to power outages and low spectral purities caused by mode hops in the master oscillator for the atmospheric backscatter and water vapor cross sections respectively. Furthermore, the instrumental and background-induced noise decreases the SNR of the measurement and limits the maximum resolvable daytime water vapor number density measurement to between 2.0 and $\sim 3 \mathrm{~km}$.

Wide spatial and temporal variabilities in both the atmospheric backscatter and the water vapor number 
density cross sections can be seen over the 60 -h period. Two collocated radiosonde measurements indicated by the black dotted lines in Fig. 10b were made to assess the daytime and nighttime performances of the DIAL instrument relative to an in situ measurement given a relatively clean atmosphere. The first radiosonde measurement launched at 1435 UTC (0835 MDT) is presented in Fig. 9a. As previously discussed, relatively good agreement between the two daytime measurements can be seen from $0.5 \mathrm{~km}$ up to the top of the boundary layer. The strong shearing layer caused by the westerly winds diminishes at approximately 1700 UTC (1100 MDT) as the wind direction measured by a collocated weather station suddenly changed from SE to N, causing the boundary layer and the associated aerosol backscatter and water vapor content to drop to approximately $1.75 \mathrm{~km}$. Another shift in the wind direction from $\mathrm{N}$ to $\mathrm{E}$ occurred at approximately 2100 UTC (1500 MDT), causing noticeable convective turbulence in both the atmospheric backscatter and water vapor number density cross sections. The strong westerly winds continued until 1900 UTC (1300 MDT) on 13 September 2009 when a low pressure system reached the valley.

Because of the presence of strong northwesterly winds, the valley surrounding the local area suffered from minimal aerosol loading, which resulted in average optical depths of 0.036 and 0.024 as measured by the CIMEL 318 solar radiometer for 11 and 12 September 2009 , respectively. As a result of the low optical thicknesses measured throughout the dataset, longer integration periods were required to achieve desirable SNRs. Consequently, less scattering in the boundary layer was observed and atmospheric backscatter and water vapor number density cross sections could be obtained up to higher altitudes, reaching well into the free troposphere at the cost of longer integration periods. A plot of the background subtracted counts as a function of altitude for a 30 -min total integration period (15 min sideline, 15 min offline) centered around 0700 UTC (0100 MDT) on 12 September 2009 is shown in Fig. 11. The dashed and solid lines represent the sideline and offline atmospheric backscattered counts measured by the DIAL instrument, respectively. Once again, a large dynamic range of $\sim 10^{4}$ from initial overlap at $0.3-0.5 \mathrm{~km}$ up to $6.0 \mathrm{~km}$ can be seen in the backscattered returns for the sideline wavelength where the attenuation relative to the offline wavelength is attributed to water vapor absorption. Good SNRs can be seen from near the surface up to $6.0 \mathrm{~km}$ due to the lack of aerosol loading in the boundary, resulting in more laser power being available for scattering in the free troposphere. A thin aerosol layer at $2.5 \mathrm{~km}$ corresponding to the approximate altitude of the top of the shearing layer from the previous

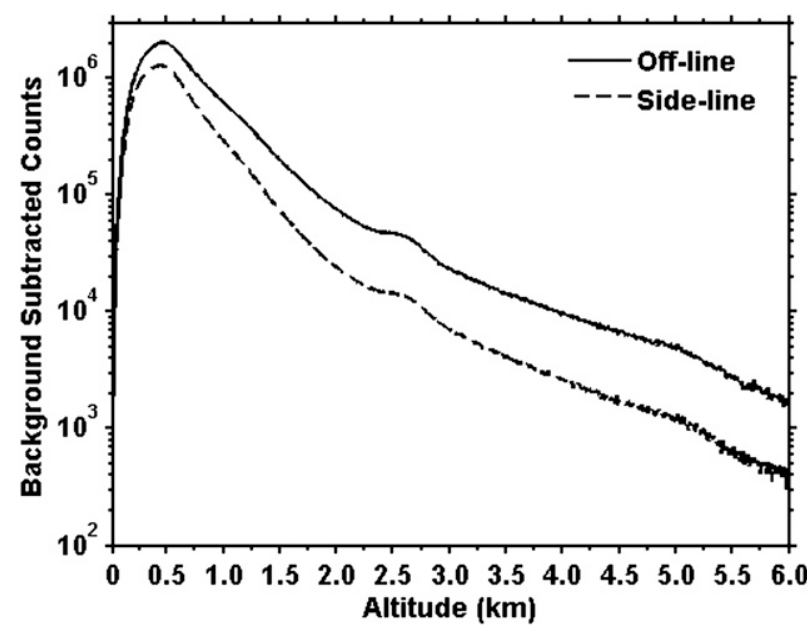

FIG. 11. Log-linear plot of nighttime background-subtracted sideline and offline atmospheric backscattered returns for a $30-\mathrm{min}$ (15 min sideline and 15 min offline) total integration period centered around 0700 UTC (0100 MDT) 12 Sep 2009. A 7.5-m-range resolution and a 30-min total integration period yield a high SNR of the backscattered returns beyond the boundary layer well into the free troposphere. An optically thin aerosol layer can be seen at approximately $2.5 \mathrm{~km}$.

day is visible in both the sideline and offline returns. A water vapor number density profile calculated using the backscattered returns from Fig. 11 is compared to an in situ collocated radiosonde measurement as indicated by the black dashed line in Fig. 10b at 0700 UTC and is shown in Fig. 12. Good agreement between the two measurements from 0.5 up to $4.5 \mathrm{~km}$ can be seen using 150-m-averaged range bins and a 30-min total integration period. A relatively weak temperature inversion at the $2.5-\mathrm{km}$ shearing layer can be seen transporting a thin layer of aerosols. The warmer aerosol-loaded layer resulted in a capping inversion where an $\mathrm{RH}$ of $2.6 \%$ was calculated using a radiosonde-derived temperature profile. Below, a stable nocturnal boundary layer can be seen throughout the evening in Fig. 10, where very little mixing of the boundary occurs the following day. Preceding a low pressure front, convective mixing of boundary layer aerosols and water vapor can be seen in Figs. 10a and 10b, respectively, starting at approximately 2000 UTC (1400 MDT) on 12 September 2009. The boundary layer water vapor content then begins to further increase rapidly as the front fully moves into the valley at approximately 0600 UTC (0000 MDT) on 13 September 2009. Appreciable cloud cover starting at approximately 2000 UTC (1400 MDT) on 13 September 2009 limited the maximum resolvable attitude for water vapor measurements to between 2.0 and $2.5 \mathrm{~km}$ due to the increase in spectral brightness of the background noise induced by the nonselective scattering of the cumulus clouds. 


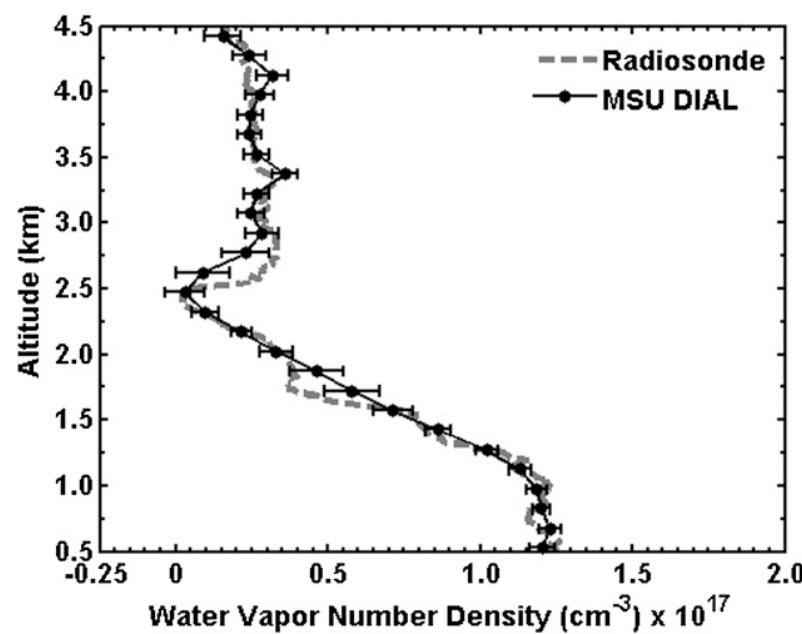

FIG. 12. Vertical water vapor number density profile recorded starting at 0645 UTC (1245 MDT) 12 Sep 2009 using the atmospheric returns obtained from Fig. 11. A 30-min total integration period and 150-m down-sampled vertical range resolution are displayed, yielding good agreement between the water vapor DIAL measurements and the collocated in situ measurements from 0.5 up to $4.5 \mathrm{~km}$.

\section{Discussion}

Daytime and nighttime water vapor number density cross sections have been recorded using relatively short integration periods with minimal operator interaction. Currently, the largest drawback of the MSU DIAL instrument for long-term boundary layer water vapor monitoring is the limited daytime maximum resolvable altitude for water vapor retrievals. Errors in the daytime measurement arise from both random and systematic sources. Systematic errors such as the laser linewidth, spectral purity, and water vapor line selection criteria have been discussed in detail in Nehrir et al. (2009). The major sources of error in the DIAL measurements are induced by random errors and are the most difficult to quantify. Inherent dark noise induced by the DIAL receiver's detector and noise in the background signal are the two main sources of random error.

Using the Moderate Resolution Atmospheric Transmission (MODTRAN) database, the daylight background radiation levels at a sun zenith angle of $35^{\circ}$ were simulated to better understand the daylight background signal levels relative to the transmitter pulse energy and its affects on the APD detector. For a summer atmospheric model for a midlatitude region, an average atmospheric radiation of $0.058 \mathrm{~W} \mathrm{~cm} \mathrm{cr}^{-2} \mathrm{sr}^{-1}$ was calculated. A background photon count measurement using the DIAL receiver for a sun zenith elevation similar to that of the simulation resulted in an average of 1400 background counts per 20000 laser shots, yielding $1.4 \mathrm{P}_{\mathrm{e}}$ (photoelectrons) $\mu \mathrm{s}^{-1}$. This is well below the saturation point of the detector, but it is at the upper bound of the linear operating region of the APD and requires a linearity correction factor of approximately 1.1 during postprocessing. Although the APD performance is not directly affected by the solar background noise, the variability in the shot-to-shot background noise has a large affect on the DIAL calculation, especially for low-energy DIAL instruments. To account for the shot-to-shot variability in the background noise, two approaches can be used. A longer dead-time integration period prior to the pulse and a second narrowband optical filter with a high out-of-band rejection ratio in the optical receiver can be implemented to decrease the susceptibility to background noise.

The performance of the DIAL instrument is highly dependent on the composition of the planetary boundary layer. Given a clear continental air atmosphere with low aerosol optical depths, the background noise can be significantly decreased due to the lack of multiple forward scattering from the sun, but will also require the need for longer integration periods due to the lack of Mie scattering in the atmosphere. Conversely, high optical depths resulting from aerosol loading can increase the background noise level at the DIAL receiver while requiring less integration time of the backscattered returns. Examples of DIAL-backscattered returns for relatively high (0.071) and low (0.024) aerosol optical depths (AODs) measured by the CIMEL 318 can be seen in Figs. 5 and 11, respectively. A decrease in the AOD by a factor of $\sim 3$ from 0.071 to 0.024 increased the maximum resolvable altitude for water vapor number density measurements from 3.8 to $4.5 \mathrm{~km}$ while increasing the required total integration periods to achieve acceptable SNRs from 20 to $30 \mathrm{~min}$. Thus, there is an inverse relationship between both the AOD of the atmosphere and the total integration period required for the DIAL measurement, as well as between the AOD and the maximum resolvable altitude for water vapor measurements. Hence, for a given atmospheric condition, a tradeoff between the maximum altitude, the spatial resolution, as well as the temporal resolution of the DIAL measurement is necessary such that an optimal SNR can be achieved.

The moderate 20-30-min integration period required to achieve reasonable SNRs of the backscattered returns ultimately limits the utility of the micropulse DIAL instrument for near-term weather predictions as well as measurements in a turbulent atmosphere. Currently, the second-generation DIAL instrument has the capability of monitoring prominent tropospheric water vapor processes under varying boundary layer conditions. Total integration periods approaching $1-5 \mathrm{~min}$ will be required 
to resolve short-lived water vapor dynamics in both static and turbulent fields.

The second-generation DIAL instrument can be improved by addressing three major issues, including increasing the laser transmitter pulse energy; decreasing the switching time between the online, sideline, and offline wavelengths; and packaging (mechanical vibration and temperature compensation) the DIAL instrument for field deployment. A third-generation water vapor DIAL instrument being developed at Montana State University has been designed to address all three major limiting factors mentioned above. The basis of the third-generation DIAL instrument is focused around two custom-built high-power ECDLs and fiber optic electromechanical switches to increase the output power, decrease the online and offline switching time and overall integration period, and to minimize the optical footprint.

\section{Conclusions}

A second-generation diode-laser-based DIAL instrument for water vapor profiling in the lower troposphere has been built, tested, and has consistently measured both nighttime and daytime water vapor number density profiles up to the top of the planetary boundary layer that compare favorably with collocated radiosonde measurements. Nighttime and daytime atmospheric backscatter and water vapor number density cross sections have been recorded over extended periods using 2030-min total integration periods where a profile interpolation method provided up-sampled spatial and temporal resolutions for viewing purposes. This is believed to be the first demonstration of a daytime operating diode-laser-based water vapor DIAL instrument. The laser transmitter is based on an actively pulsed, cascaded, widely tunable master ECDL oscillator semiconductor optical power amplifier configuration producing up to $1-2 \mu \mathrm{J}$ pulses. The DIAL transmitter is capable of accessing any water vapor absorption line in the 824 841-nm spectral region, an important capability that has been called for in other studies. Despite the low-power limitations of the water vapor DIAL instrument, a narrow field of view, high pulse repetition frequencies, and moderate averaging times have provided sufficient Mie and Rayleigh backscattered returns for water vapor profiling up through the top of the boundary layer and cloud and aerosol profiling well into the free troposphere. Nighttime and daytime tropospheric water vapor number density profiles and time-height plots have been measured using sideline tuning of the second-generation DIAL instrument with 20-30-min averaging times, resulting in $5 \%-15 \%$ errors of the water vapor molecular density measurement when compared to an in situ collocated radiosonde measurement. The performance of the eye-safe DIAL transmitter coupled with the extremely compact footprint of the second- and thirdgeneration DIAL instruments, along with the exceptionally high wall plug efficiencies of diode-laser-based technologies, have given the diode-laser-based DIAL instrument developed at Montana State University the potential to be deployed for use in ground-based multipoint water vapor lidar networks for climate monitoring and weather forecasting applications.

Acknowledgments. The authors thank the two reviewers for their detailed reviews that improved the quality of this manuscript. This work was supported by NASA EPSCoR under Grant NNX08AT69A and the NASA Graduate Student Researchers Program (GSRP) under Grant NNX08AR90H.

\section{REFERENCES}

ANSI, 1993: American National Standard for Safe Use of Laser. Z136.1-1993, American National Standards Institute.

Bosenberg, J., 1998: Ground based-differential absorption lidar for water vapor and temperature profiling: Methodology. Appl. Opt., 37, 3845-3860.

Browell, E. V., S. Ismail, and B. E. Grossman, 1991: Temperature sensitivity of differential absorption lidar measurements of water vapor in the $720 \mathrm{~nm}$ region. Appl. Opt., 30, 1517-1524.

,-- , and W. B. Grant, 1998: Differential absorption lidar (DIAL) measurements from air and space. Appl. Phys., 67B, 399-410.

Charlson, R. J., S. E. Schwartz, J. M. Hales, R. D. Cess, J. A. Coakley Jr., J. E. Hensen, and D. J. Hofmann, 1992: Climate forcing by anthropogenic aerosols. Science, 255, 423-430.

Ehret, G., C. Kiemle, W. Renger, and G. Simmet, 1993: Airborne remote sensing of tropospheric water vapor with a near-infrared differential absorption lidar system. Appl. Opt., 32, 4534-4551.

— A. Fix, V. Weiss, G. Poberaj, and T. Baumert, 1998: Diode laser seeded parametric oscillator for airborne water vapor DIAL applications in the upper troposphere and lower stratosphere. Appl. Phys., 67B, 427-431.

Eichinger, W. E., P. R. Forman, J. Griegos, M. A. Osborn, D. Richter, L. L. Tellier, and R. Thornton, 1999: The development of a scanning Raman water vapor lidar for boundary layer and tropospheric observations. J. Atmos. Oceanic Technol., 16, 17531766.

Forster, P., and Coauthors, 2007: Changes in atmospheric constituents and in radiative forcing. Climate Change 2007: The Physical Science Basis, S. Solomon et al., Eds., Cambridge University Press, 747-845.

Goldsmith, J. E. M., F. H. Blair, S. E. Bisson, and D. D. Turner, 1998: Turn key Raman lidar for profiling atmospheric water vapor, clouds, and aerosols. Appl. Opt., 37, 4979-4990.

Harms, J., W. Lahmann, and C. Weitkamp, 1978: Geometrical compression of lidar return signals. Appl. Opt., 17, 1131-1135.

Ismail, S., E. V. Browell, R. A. Ferrare, S. A. Kooi, M. B. Clayton, V. G. Brackett, and P. B. Russell, 2000: LASE measurements of aerosol and water vapor profiles during TARFOX. J. Geophys. Res., 105, 9903-9916. 
Kiehl, J. T., and K. E. Trenberth, 1997: Earth's annual global mean energy budget. Bull. Amer. Meteor. Soc., 78, 197-208.

Kovalev, V. A., and W. E. Eichinger, 2004: Elastic Lidar: Theory, Practice, and Analysis Methods. John Wiley and Sons, 615 pp.

Machol, J. L., and Coauthors, 2004: Preliminary measurements with an automated compact differential absorption lidar for the profiling of water vapor. Appl. Opt., 43, 3110-3121.

Nehrir, A. R., K. S. Repasky, J. L. Carlsten, M. D. Obland, and J. A. Shaw, 2009: Water vapor profiling using a widely tunable, amplified diode laser based differential absorption lidar (DIAL). J. Atmos. Oceanic Technol., 26, 733-745.

Rall, J. A. R., J. B. Abshire, D. Reusser, and M. Humphrey, 1996: Measurements of atmospheric water vapor using a compact AlGaAs laser based DIAL instrument. Conf. on Lasers and Electro-Optics, Anaheim, CA, Optical Society of America, Paper CWD5.

Reagan, J. A., T. W. Cooley, and J. A. Shaw, 1993: Prospects for an economical, eye safe water vapor lidar. Proc. Int. Geoscience and Remote Sensing Symp., Tokyo, Japan, IEEE, 872-874.

Takase, K., J. K. Stockton, and M. A. Kasevich, 2007: High-power pulsed-current mode operation of an overdriven tapered amplifier. Opt. Lett., 32, 2617-2619.

Trenberth, K. E., and Coauthors, 2007: Observations: Surface and atmospheric climate change. Climate Change 2007: The Physical Science Basis, S. Solomon et al., Eds., Cambridge University Press, 747-845.
Whiteman, D. N., S. H. Melfi, and R. A. Ferrare, 1998: Raman lidar system for the measurement of water vapor and aerosols in the earth's atmosphere. Appl. Opt., 31, 3068-3082.

Wirth, M., A. Fix, P. Mahnke, H. Schwarzer, F. Schrandt, and G. Ehret, 2009: The airborne multi-wavelength water vapor differential absorption lidar WALES: System design and performance. Appl. Phys., 96B, 201-213.

Wulfmeyer, V., 1998: Ground-based differential absorption lidar for water vapor and temperature profiling: Development and specifications of a high performance laser transmitter. Appl. Opt., 37, 3804-3824.

—_, and J. Bosenberg, 1998: Ground-based differential absorption lidar for water vapor profiling: Assessment of accuracy, resolution, and meteorological applications. Appl. Opt., 37, $3825-3844$

— , and G. Feingold, 2000: On the relationship between relative humidity and particle backscatter coefficient in the marine boundary layer determined with differential absorption lidar. J. Geophys. Res., 105, 4729-4741.

— and airborne water vapor differential absorption lidar: I. Overview and theory. Appl. Opt., 40, 5304-5320.

Xiong, Y., S. Murphy, J. L. Carlsten, and K. S. Repasky, 2006: Design and characteristics of a tapered amplifier diode system by seeding with continuous-wave and mode locked external cavity diode laser. Opt. Eng., 45, 124205. 